\title{
ON THE FORMAL SOLUTION OF DUAL INTEGRAL EQUATIONS ${ }^{1}$
}

R. K. SAXENA

1. Introduction. The object of the present paper is to obtain a formal solution of certain dual integral equations possessing general $H$-functions as kernels. The result obtained is in fact an extension of the result given quite recently by Fox $[3$, p. 395]. The importance of the result established lies in the fact that the solution of dual integral equations involving general Meijer's $G$-functions and nearly all the special functions occurring in Applied Mathematics can be derived as particular cases from our result on account of the most general character of the $H$-function.

We define the $H$-function by means of the Mellin-Barnes integral [2, p. 49]

$$
B_{p+n, q+m}^{m, n}\left(x \mid \begin{array}{l}
a_{j}, A_{j} \\
b_{k}, B_{k}
\end{array}\right)
$$

$$
\begin{aligned}
& =H_{p+n, q+m}^{m, n}\left(x \mid \begin{array}{l}
a_{1}, A_{1}, a_{2}, A_{2}, \cdots, a_{n+p}, A_{n+p} \\
b_{1}, B_{1}, b_{2}, B_{2}, \cdots, b_{m+q}, B_{m+q}
\end{array}\right) \\
& =\frac{1}{2 \pi i} \int_{L} \frac{\prod_{j=1}^{m} \Gamma\left(b_{j}+B_{j} s\right) \prod_{j=1}^{n} \Gamma\left(a_{j}-A_{j} s\right) x^{-\bullet} d s}{\prod_{j=1}^{q} \Gamma\left(b_{m+j}-B_{m+j} s\right) \prod_{j=1}^{p} \Gamma\left(a_{n+j}+A_{n+j} s\right)}
\end{aligned}
$$

where an empty product is to be interpreted as unity and the following simplified assumptions are made.

(i) $a_{j}, A_{j}, b_{k}$ and $B_{k}$ are all real for $j=1,2, \cdots,(p+n)$ and $k=1,2$, ..., $(q+m)$.

(ii) $A_{j}$ 's and $B_{k}$ 's are positive for $j=1,2, \cdots,(p+n)$ and $k=1,2$, ..., $(q+m)$.

(iii) All the poles of the integrand in (2) are simple.

(iv) Let $s=\sigma+i t, \sigma$ and $t$ being real; then the contour $L$ along which the integral of (2) is taken, is a straight line parallel to the imaginary axis in the complex $s$-plane whose equation is $\sigma=\sigma_{0}$, where $\sigma_{0}$ is a constant. The contour is such that all the poles of $\Gamma\left(b_{k}+B_{k} s\right)$

Received by the editors April 6, 1966.

1 Research supported by a Post-doctoral Fellowship of the National Research Council of Canada. 
for $k=1,2, \cdots, m$ lie to the left and those of $\Gamma\left(a_{j}-A_{j} s\right)$ for $j=1,2$, $\cdots, n$, to the right of it.

(v) $\sum_{j=1}^{n} A_{j}+\sum_{j=1}^{m} B_{j}=\sum_{j=1}^{p} A_{n+j}+\sum_{j=1}^{q} B_{m+j}$.

(vi) $\quad \lambda=-\sum_{j=1}^{m} B_{j}+\sum_{j=1}^{n} A_{j}-\sum_{j=1}^{q} B_{m+j}+\sum_{j=1}^{p} A_{n+j}$.

(vii) $\quad \mu=\sum_{j=1}^{n} a_{j}+\sum_{j=1}^{m} b_{j}-\sum_{j=1}^{p} a_{n+j}-\sum_{j=1}^{q} b_{m+j}+\frac{1}{2}(p+q-m-n)$.

(viii) $x>0$.

(ix) $\lambda \sigma_{0}+\mu+1<0$.

It follows from the asymptotic representation of the gamma function $[1$, p. 47$]$

$$
\lim _{|t| \rightarrow \infty}|\Gamma(\sigma+i t)||t|^{1 / 2-\sigma} \exp \left(\frac{\pi}{2}|t|\right)=(2 \pi)^{1 / 2}
$$

on setting $s=\sigma_{0}+i t, x=\operatorname{Re}^{i \phi}(R>0, \phi$ real $)$ that the absolute value of the integrand of $(2)$ is comparable with $(\rho / R)^{\sigma_{0}}|t|^{\lambda \sigma_{0}+\mu} e^{\phi t}$ where

$$
\rho=\prod_{j=1}^{n}\left(A_{j}\right)^{A_{j}} \prod_{j=1}^{m}\left(B_{j}\right)^{-B_{j}} \prod_{j=1}^{p}\left(A_{n+j}\right)^{A_{n+j}} \prod_{j=1}^{q}\left(B_{n+j}\right)^{-B_{n+j}},
$$

when $|t|$ is large and therefore the integral (2) converges absolutely if the conditions (viii) and (ix) are satisfied.

The dual integral equations to be discussed here are as follows:

$$
\begin{aligned}
& \int_{0}^{\infty} H_{p+n, q+m}^{m, n}\left(x u \mid \begin{array}{l}
a_{j}, A_{j} \\
b_{k}, B_{k}
\end{array}\right) f(u) d u=\phi(x), \quad 0<x<1, \\
& \int_{0}^{\infty} H_{p+n, q+m}^{m, n}\left(x u \mid \begin{array}{l}
c_{j}, A_{j} \\
d_{k}, B_{k}
\end{array}\right) f(u) d u=\psi(x), \quad x>1,
\end{aligned}
$$

where $\phi(x)$ and $\psi(x)$ are given and $f(x)$ is to be determined. We assume that the $H$-function of (5) satisfies all the conditions given above with $a_{j}$ replaced by $c_{j}$ and $b_{k}$ replaced by $d_{k}$ for $j=1,2, \cdots,(n+p)$ and $k=1,2, \cdots,(q+m)$. We also assume that a common value of $\sigma_{0}$ can be found for both the $H$-functions.

2. The Mellin transform. As usual we denote the Mellin transform of $f(u)$ by $\mathfrak{M}\{f(u)\}$ and if $\mathfrak{M}\{f(u)\}=F(s)$, we shall also write $f(u)$ $=\mathfrak{M}^{-1}\{F(s)\}$. 
Formally we have

$$
\mathfrak{M}\{f(u)\}=F(s)=\int_{0}^{\infty} u^{s-1} f(u) d u
$$

and

$$
\mathfrak{M}^{-1}\{F(s)\}=f(u)=\frac{1}{2 \pi i} \int_{L} F(s) u^{-8} d s .
$$

Conditions of the validity of (6) and (7) are given in $[6, \S \S 1.29$, 1.37].

The Parseval theorem of the Mellin transform has been restated by Fox [3, p. 391] in the following form which will be found useful in our investigations.

If

$$
\mathfrak{M}\{h(u)\}=H(s)
$$

and

$$
\mathfrak{M}\{f(x u)\}=x^{-s} F(s),
$$

then

$$
\int_{0}^{\infty} h(x u) f(u) d u=\frac{1}{2 \pi i} \int_{L} x^{-s} H(s) F(1-s) d s .
$$

From (2) and (7) it is easily seen that

$$
\mathfrak{M}\left[H_{p+n, q+m}^{m, n}\left(x \mid \begin{array}{l}
a_{j}, A_{j} \\
b_{k}, B_{k}
\end{array}\right)\right]
$$

$$
=\frac{\prod_{j=1}^{m} \Gamma\left(b_{j}+s B_{j}\right) \prod_{j=1}^{n} \Gamma\left(a_{j}-s A_{j}\right)}{\prod_{j=1}^{q} \Gamma\left(b_{m+j}-s B_{m+j}\right) \prod_{j=1}^{p} \Gamma\left(a_{n+j}+s A_{n+j}\right)}
$$

and this follows from (2) if the condition (ix) of $\$ 1$ holds.

On using $\mathfrak{M}\{f(u)\}=F(s)$ and applying (8) to (4) and (5) we find that

$$
\frac{1}{2 \pi i} \int_{L} \frac{\prod_{j=1}^{m} \Gamma\left(b_{j}+s B_{j}\right) \prod_{j=1}^{n} \Gamma\left(a_{j}-s A_{j}\right) x^{-s} F(1-s) d s}{\prod_{j=1}^{q} \Gamma\left(b_{m+j}-s B_{m+j}\right) \prod_{j=1}^{p} \Gamma\left(a_{n+j}+s A_{n+j}\right)}=\phi(x),
$$

where $0<x<1$ and 
(11)

$$
\frac{1}{2 \pi i} \int_{L} \frac{\prod_{j=1}^{m} \Gamma\left(d_{j}+s B_{j}\right) \prod_{j=1}^{n} \Gamma\left(c_{j}-s A_{\jmath}\right) x^{-s} F(1-s)}{\prod_{j=1}^{q} \Gamma\left(d_{j+m}-s B_{m+j}\right) \prod_{j=1}^{p} \Gamma\left(c_{j+n}+s A_{n+j}\right)}=\psi(x)
$$

where $x>1$ by virtue of (9).

3. The reduction of (10) and (11) to equations with a common kernel. In this section we will transform the equations (10) and (11) in to two others with the same kernel by the application of fractional integration operators. Our main aim here is to transform

$$
\frac{\prod_{j=1}^{n} \Gamma\left(a_{j}-s A_{j}\right)}{\prod_{j=1}^{q}\left(b_{m+j}-s B_{m+j}\right)} \text { of (10) into } \frac{\prod_{j=1}^{n} \Gamma\left(c_{j}-s A_{j}\right)}{\prod_{j=1}^{q} \Gamma\left(d_{m+j}-s B_{m+j}\right)}
$$

of (11) and

$$
\frac{\prod_{j=1}^{m} \Gamma\left(d_{j}+s B_{j}\right)}{\prod_{j=1}^{p} \Gamma\left(c_{n+j}+A_{n+j} s\right)} \text { of (11) into } \frac{\prod_{j=1}^{m} \Gamma\left(b_{j}+s B_{j}\right)}{\prod_{j=1}^{p} \Gamma\left(a_{n+j}+s A_{n+j}\right)}
$$

of (10).

In making these transformations we will make use of the fractional integration operators defined by Erdélyi [1, p. 220].

$$
\begin{aligned}
& \tau[\alpha, \beta: r: w(x)]=\{r / \Gamma(\alpha)\} \bar{x}^{r \alpha+r-\beta-1} \int_{0}^{x}\left(x^{r}-v^{r}\right)^{\alpha-1} v^{\beta} w(v) d v \\
& \Re[\alpha, \beta: r: w(x)]=\{r / \Gamma(\alpha)\} x^{\beta} \int_{x}^{\infty}\left(v^{r}-x^{r}\right)^{\alpha-1} v^{-\beta-r \alpha+r-1} w(v) d v .
\end{aligned}
$$

When $r=1$, (14) and (15) reduce to Kober's operators [4, p. 194]. Recently Fox has shown that there is no essential difference between the two operators [3, p. 393]. Both the operators $\tau$ and $\Re$ exist provided $w(x) \in L_{p}^{\prime}(0, \infty), p^{\prime} \geqq 1, \alpha>0, \beta>\left(1-p^{\prime}\right) / p^{\prime}[1, \S 2]$. If in addition $w(x)$ can be differentiated sufficiently of ten, then the operators $\tau$ and $\Re$ exist for negative as well as positive values of $\alpha[1, \S 2$, p. 222].

In the contracted forms we write 


$$
\begin{array}{ll}
\text { (16) } & \tau\left[\left(a_{j}-c_{j}\right), c_{j} A_{j}^{-1}-1: A_{j}^{-1}: w(x)\right]=\tau_{j}[w(x)], \\
\text { (17) } & \tau\left[\left(d_{m+k}-b_{m+k}\right),\left(b_{m+k} B_{m+k}^{-1}-1\right): B_{m+k}^{-1}: w(x)\right]=\tau_{k}^{*}[w(x)], \\
\text { (18) } & \Re\left[\left(d_{l}-b_{l}\right), b_{l} B{ }^{-1}: B_{l}^{-1}: w(x)\right]=\Re_{l}[w(x)], \\
\text { (19) } & \Re\left[\left(a_{n+h}-c_{n+h}\right), c_{n+h} A_{n+h}^{-1}: A_{n+h}^{-1}: w(x)\right]=\Re_{h}^{*}[w(x)] .
\end{array}
$$

We now proceed to make the first transformation.

In (10) replace $x$ by $v$, multiply by $v^{c_{n} e_{n}-1}\left(x^{e_{n}}-v^{e_{n}}\right)^{a_{n}-c_{n}-1}$ where $e_{n}=1 / A_{n}$, integrate through the integral sign with respect to $v$ from 0 to $x, 0<x<1$ and apply the well-known Beta function formula, we then obtain $[3, \S 3]$

$$
\begin{gathered}
\frac{1}{2 \pi i} \int_{L} \frac{\prod_{j=1}^{m} \Gamma\left(b_{j}+B_{j} s\right) \prod_{j=1}^{n-1} \Gamma\left(a_{j}-A_{j} s\right) \Gamma\left(c_{n}-A_{n} s\right)}{\prod_{j=1}^{q} \Gamma\left(b_{m+j}-s B_{m+j}\right) \prod_{j=1}^{p} \Gamma\left(a_{n+j}+A_{n+j} s\right)} x^{-s} F(1-s) d s \\
=\frac{e_{n x} e_{n-a_{n} e_{n}}}{\Gamma\left(a_{n}-c_{n}\right)} \int_{0}^{x} v^{e_{n} c_{n}-1}\left(x^{e_{n}}-v^{e_{n}}\right)^{a_{n}-c_{n}-1} \phi(v) d v=\tau_{n}[\phi(v)]
\end{gathered}
$$

where $0<x<1$ and $e_{n}=1 / A_{n}$, by virtue of (14) and (16).

On transforming (20) successively, for $k=(n-1),(n-2), \cdots$, $3,2,1$ by the application of the operators $\tau_{k}$ and by $\tau_{j}^{*}$ for $j=q, q-1$, $\cdots, 3,2,1$ we get finally

$$
\begin{aligned}
\frac{1}{2 \pi i} \int_{L} \frac{\prod_{j=1}^{m} \Gamma\left(b_{j}+B_{j} s\right) \prod_{j=1}^{n} \Gamma\left(c_{j}-s A_{j}\right) x^{-s} F(1-s) d s}{\prod_{j=1}^{q} \Gamma\left(d_{m_{+} j}-s B_{m+j}\right) \prod_{j=1}^{p} \Gamma\left(a_{n+j}+s A_{n+j}\right)} \\
\quad=\tau_{1}^{*}\left[\tau_{2}^{*} \cdots \tau_{q}^{*} \tau_{1} \cdots \tau_{n}[\phi(x)] \cdots\right] \text { where } 0<x<1
\end{aligned}
$$

In a similar manner by the application of the operators $\Re_{l}$ and $\Re_{h}^{*}$ given in (18) and (19) respectively for $l=m, m-1, \cdots, 3,2,1$ and $h=p, p-1, \cdots, 3,2,1$ to (11) it can be easily seen that it transforms in to the desired form

$$
\begin{aligned}
\frac{1}{2 \pi i} \int_{L} \frac{\prod_{j=1}^{m} \Gamma\left(b_{j}+B_{j} s\right) \prod_{j=1}^{n} \Gamma\left(c_{j}-s A_{j}\right) x^{-8} F(1-s) d s}{\prod_{j=1}^{q} \Gamma\left(d_{m+j}-s B_{m+j}\right) \prod_{j=1}^{p} \Gamma\left(a_{n+j}+s A_{n+j}\right)} \\
\left.\quad=\Re_{1}^{*} \mid \Re_{2}^{*} \cdots \Re_{p}^{*} \Re_{1} \cdots \Re_{m}[\psi(x)] \cdots\right], \text { where } x>1
\end{aligned}
$$


On setting

$$
\begin{aligned}
& t(x)=\stackrel{*}{\tau_{1}}\left[\tau_{2}^{*} \cdots{ }_{\tau_{q}}^{*} \tau_{1} \tau_{2} \cdots \tau_{n}[\phi(x)] \cdots\right], \quad 0<x<1 \\
& =\Re_{1}^{*}\left[\Re_{2}^{*} \cdots \Re_{p}^{*} \Re_{1} \Re_{2} \cdots \Re_{m}[\psi(x)] \cdots\right], \quad x>1
\end{aligned}
$$

(21) and (22) can be put in to a compact form

$$
\begin{aligned}
\frac{1}{2 \pi i} \int_{L} \frac{\prod_{j=1}^{m} \Gamma\left(b_{j}+s B_{j}\right) \prod_{j=1}^{n} \Gamma\left(c_{j}-s A_{j}\right)}{\prod_{j=1}^{q} \Gamma\left(d_{m+j}-s B_{m+j}\right) \prod_{j=1}^{p} \Gamma\left(a_{n+j}+s A_{n+j}\right)} x^{-8} F(1-s) d s \\
\quad=t(x) .
\end{aligned}
$$

(24) is the reduction of (10) and (11) to two equations with a common kernel. On treating the kernel of (24) as an unsymmetrical Fourier kernel and following a procedure adopted by Fox [3, p. 395] it is found that (24) can be written as

$$
\begin{aligned}
f(x)= & \frac{1}{2 \pi i} \int_{L} \frac{\prod_{j=1}^{q} \Gamma\left(d_{m+j}+s B_{m+j}-B_{m+j}\right) \prod_{j=1}^{p} \Gamma\left(a_{n+j}+A_{n+j}-s A_{n+j}\right)}{\prod_{j=1}^{m} \Gamma\left(b_{j}+B_{j}-B_{j} s\right) \prod_{j=1}^{n} \Gamma\left(c_{j}-A_{j}+s A_{j}\right)} \\
& \times x^{-8} T(1-s) d s
\end{aligned}
$$

where $\mathfrak{M}\{t(x)\}=T(s)$.

This is the formal solution of (10) and (11) and many important properties of the function $f(x)$ can be obtained from it. Applying (8) to (25) we see that

$$
f(x)=\int_{0}^{\infty} H_{p+n, q+m}^{q, p}
$$

$$
\cdot\left(x u \mid \begin{array}{l}
a_{n+j}+A_{n+j}, A_{n+j}, c_{j}-A_{j}, A_{j} \\
d_{m+k}-B_{m+k}, B_{m+k}, b_{k}+B_{k}, B_{k}
\end{array}\right) t(u) d u .
$$

It is clear from the nature of this solution that it can be written down by inspection from (4) and (5). Since our method is formal it does not give the conditions of validity of the solution. The solution (26) can be verified by reversing all the steps from (4), (5) to (26) by means of the operators $\tau^{-1}$ and $\Re^{-1}$ defined by Fox [3, p. 397]

$$
\begin{aligned}
\tau^{-1}[\alpha, \beta ; r: f(x)] & =\tau[-\alpha, \beta+r \alpha: r: f(x)], \\
\Re^{-1}[\alpha, \beta: r: f(x)] & =\Re[-\alpha, \beta+r \alpha: r: f(x)]
\end{aligned}
$$


and establishing conditions which justify these reversals. The $H$ function of (26) exists if $\lambda \sigma_{0}+\mu^{\prime}+\lambda+1<0$, where $\mu^{\prime}$ is obtained from $\mu$ by interchanging $a_{j}$ 's by $-c_{j}$ 's, $j=1,2, \cdots, n ; b_{j}$ 's by $-b_{j}$ 's for $j=1,2, \cdots, m ; a_{n+j}$ by $-a_{n+j}, b_{m+j}$ by $-d_{m+j}$ for $j=1,2, \cdots, q$ and $\frac{1}{2}(p+q-m-n)$ by $\frac{1}{2}(m+n-p-q)$.

Here $H$-function has been used as an unsymmetrical Fourier kernel and the conditions under which $H$-functions form a pair of unsymmetrical Fourier kernel are given in $[5$, p. 357].

\section{Particular cases.}

(i) Fox's result [3, p. 395] can be derived from (26) by taking $n=p=0, q=m, B_{m+j}=B_{j}=a_{j}, b_{j}=\alpha_{j}, b_{m+j}=\beta_{j}, c_{j}=\lambda_{j}, d_{m+j}=\mu_{j}$, for $j=1,2, \cdots, m$.

(ii) On the other hand if we set $A_{j}=B_{k}=1$, for $j=1,2, \cdots$, $(p+n)$ and $k=1,2, \cdots,(m+q), H$-functions then reduce to Meijer's $G$-functions [1, p. 207] and it is seen that (4) and (5) give rise to the following dual integral equations

$$
\begin{gathered}
\int_{0}^{\infty} G_{p+n, q+m}^{m, n}\left(x u \mid \begin{array}{l}
a_{1}, \cdots, a_{n+p} \\
b_{1}, \cdots, b_{m+q}
\end{array}\right) f(u) d u=\phi(x), \quad 0<x<1, \\
\int_{0}^{\infty} G_{p+n, q+m}^{m, n}\left(x u \mid \begin{array}{l}
c_{1}, \cdots, c_{n+p} \\
d_{1}, \cdots, d_{m+q}
\end{array}\right) f(u) d u=\psi(x), \quad x>1,
\end{gathered}
$$

whose formal solution is

$$
\begin{aligned}
f(x)= & \int_{0}^{1} G_{p+n, q+m}^{q, p} \\
& \cdot\left(x u \mid \begin{array}{l}
-a_{n+1}, \cdots,-a_{n+p},-c_{1}, \cdots,-c_{n} \\
-d_{m+1}, \cdots,-d_{m+q},-b_{1}, \cdots,-b_{m}
\end{array}\right) \\
\times & \tau_{1}^{*}\left[\tau_{2}^{*} \cdots \tau_{q}^{*} \tau_{1}^{*} \cdots \tau_{n}^{*}[\phi(u)] \cdots\right] d u \\
& +\int_{1}^{\infty} G_{p+n, q+m}^{q, p} \\
& \cdot\left(x u \mid \begin{array}{l}
-a_{n+1}, \cdots,-a_{n+p},-c_{1}, \cdots,-c_{n} \\
-d_{m+1}, \cdots,-d_{m+q},-b_{1}, \cdots,-b_{m}
\end{array}\right) \\
& \times \Re_{1}^{*}\left[\Re_{2} \cdots \Re_{p} \Re_{1}^{*} \cdots \Re_{m}^{*}[\psi(u)] \cdots\right] d u
\end{aligned}
$$

by virtue of (26), and the operators are to be taken with $a_{j}$ replaced by $1-a_{j}, j=1, \cdots, n ; b_{m+j}$ by $1-b_{m+j}, j=1,2, \cdots, q ; c_{j}$ by $1-c_{j}$, $j=1,2, \cdots, n ; d_{m+j}$ by $1-d_{m+j}, j=1,2, \cdots, q B_{j}=A_{k}=1$ for 
R. K. SAXENA

$j=1,2, \cdots,(q+m)$ and $k=1,2, \cdots,(p+n)$, that is the operators will be due to Kober in this case.

In conclusion the author wishes to thank Professor Charles Fox for his helpful suggestions and criticisms during the preparation of this paper.

\section{REFERENCES}

1. A. Erdélyi, On some functional transformations, Univ. e Politec. Torino Rend. Sem. Mat. 10 (1950-1951), 217-234.

2. A. Erdélyi, et al. Higher transcendental functions, Vol. 1, McGraw-Hill, New York, 1953.

3. Charles Fox, A formal solution of certain dual integral equations, Trans. Amer. Math. Soc. 119 (1965), 389-398.

4. H. Kober, On fractional integrals and derivatives, Quart. J. Math. Oxford Ser. 11 (1940), 193-211.

5. Roop Narain, $A$ pair of unsymmetrical Fourier kernels, Trans. Amer. Math. Soc. 115 (1965), 356-369.

6. E. C. Titchmarsh, Theory of Fourier integrals, Oxford Univ. Press, Oxford, 1937. MCGILl UNIVERSITY 